\title{
A NEW RECORD OF THE GENUS CREPIDOTUS (CREPIDOTACEAE, FUNGI) FROM INDIA
}

\author{
Agretious Thomas, $\mathrm{K}^{\mathbf{1}}$. and K.P. Prasanth ${ }^{\mathbf{2}}$ \\ ${ }^{1}$ Nehru Arts and Science College, Kanhangad, Kasaragod-671314, Kerala. \\ ${ }^{2}$ Sree Narayana College, Thottada, Kannur-670007, Kerala.
}

\begin{abstract}
A Crepidotus species, $C$. cinnabarinus is recorded for the first time from India. This species grow on decaying woody plant materials. It is characterized by small to medium sized, pleurotoid, flabelliform, laterally attached basidiomata with convex to applanate pileus. Spores are generally ovoid to subglobose without a germ- pore.
\end{abstract}

Keywords: Fungi, Agaricales, Crepiditaceae, Crepidotus, new records.

\section{INTRODUCTION}

During the study of Agaric flora of Northern Kerala, we came across a species belonging to the genus Crepidotus. It was found to be a new record from India. Manjula (1983) listed five species of Crepidotus viz. C. Cystidiosus, C. eucalypticola, C. uber, C. applanatus and C. alveolus as known from India. of these, the first three were collected from Tamil Nadu state by Natarajan and Raman (1981) and later they (Natarajan and Raman, 1983) added two more species $C$. applanatus and $C$. nephrodes to the Indian records. Vrinda et al. (2000) reported two species, $C$. citrinus, and C. pezizula from Western Ghats. Mohanan (2011) added five species viz. C. calolepis, C. epicrocinus, C. grumosopilosus, C. melleus and C. reversus to the Indian records.

\section{MATERIALS AND METHODS}

Microscopic observations are made on materials mounted in 3\% aqueous KOH. Colour codes refer to Kornerup and Wanscher (1978). The collected specimen is described, illustrated and discussed. Spore statistics are based on measurement of twenty spores.

\subsection{Description of species}

Crepidotus cinnabarinus Peck, Bull. Torrey Bot. Club 22:489 (1895).

Pileus 10-45mm diameter, pleurotoid, laterally attached, flabelliform, convex to applanate when seen from the side; surface tomato red (8C8), may leach out during rain and become light orange (6A4) or greyish orange (6B4), sparsely dotted with fine, appressed squamules especially towards the margin; margin entire, at times turning fissile, initially inrolled, becoming incurved or decurved, deeply and irregularly lobate when old. Context up to $3 \mathrm{~mm}$ thick, white to orange white (5A2). Lamellae adnate, initially pale yellow (4A3) to pale orange (5A3), becoming light brown (6D4), subcrowded, up to $3 \mathrm{~mm}$ wide, with lamellulae of different lengths; edge tomato red (8C8), finely torn under a lens. Stipe 10-20 × 4-7 mm, lateral, stout, solid; surface white, tomentose at base. Odour not distinctive. Spore-print light brown (6D4).

Spores 5-8 × 4-5.5 $(6.14 \pm 0.80 \times 4.83 \pm 0.44)$ $\mu \mathrm{m}$, ovoid to subglobose, yellowish brown, thin to slightly thick walled, warty punctuate, without a germ- pore. Basidia 21.5-29 × 5.5-8 $\mu \mathrm{m}$, clavate, 4 - or sometimes 2-spored; sterigmata upto $5.5 \mu \mathrm{m}$ long. Lamella-edge sterile with crowded cheilocystidia. Cheilocystidia 13-80 × 5-14.5 $\mu \mathrm{m}$, mostly ventricosefusoid with long flexuose apices which are at times furcate, sometimes clavate, thin- or sometimes slightly thick- walled, with a light reddish brown plasmatic pigment and frequently with a fine, hyaline encrustation on the wall. Pleurocystidia scattered, occasional, similar to cheilocystidia in all aspects. Hymenophoral trama regular; hyphae $1.5-2.5 \mu \mathrm{m}$ wide, thin-walled, hyaline to pale yellow, at times finely encrusted. Pileal trama interwoven; hyphae 2$10.5 \mu \mathrm{m}$ wide, almost thin walled, light yellow to hyaline. Pileipellis a disrupted cutis; hyphae 1.5-7 $\mu \mathrm{m}$ wide, thin walled, with light reddish brown plasmatic pigment, at times with faint hyaline encrustations. Clamp-connexions absent. On the base of a decaying wood stump, in imbricate clusters, August to October. Specimens examined: India, Kerala State, Wayanad District, Ponkuzhy: 28 October 1999, A. Thomas T343; 31 October 1999, A. Thomas T343b; 25 August 2015, A. Thomas T343c. 


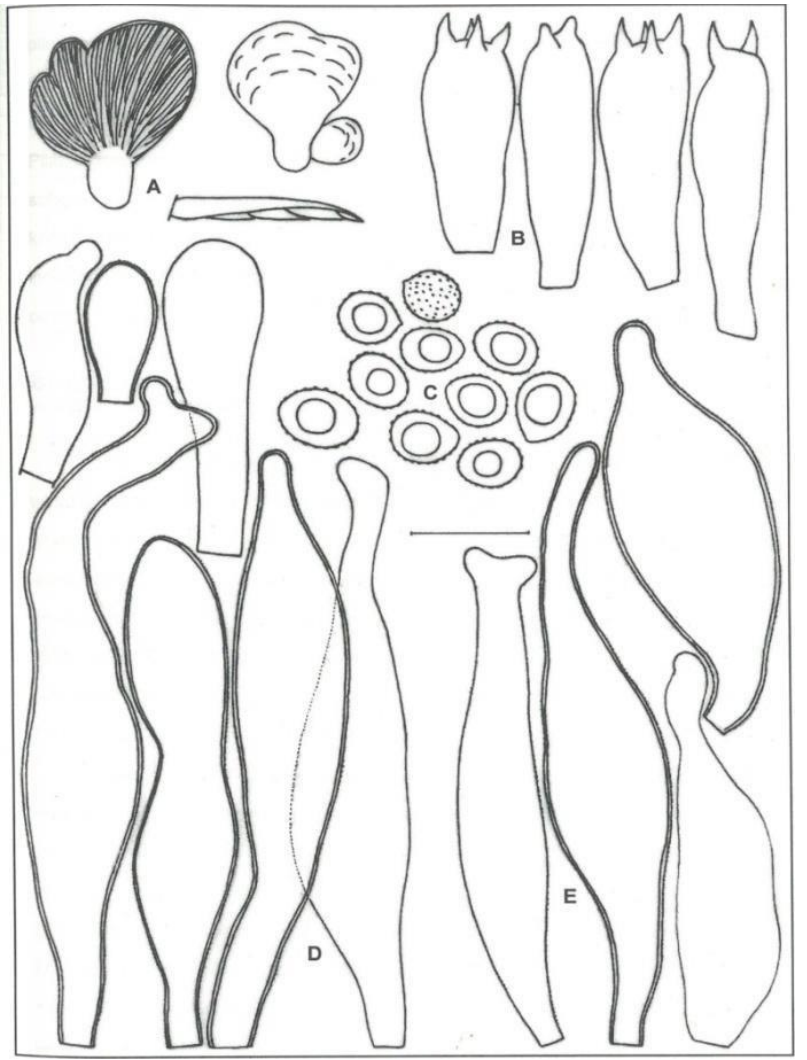

Fig. 1. Crepidotus cinnabarinus

A. Habit; B. Basidia; C. Spores; D. Cheilocystidia; E. Pleurocystidia. Scale bar $=10 \mu \mathrm{m}$

Crepidotus cinnabarinus is characterized by the distinctive tomato red colour of the pileus and lamella- edge; ovoid to subglobose, warty-punctate spores; ventricose-fusoid cheilocystidia with reddish brown plasmatic pigment; non-gelatinized trama; and absence of clamp-connexions. In Singer's (1986) system, it will come under the section Echinospori Pilat, subsection Aporpini Singer. In Hesler and Smith's (1965) classification, it is placed in subgenus Crepidotus, section Cinnabarini Hesler and Smith where only $C$. cinnabarinus is known. The Kerala collections showed some minor deviations from that species which include larger and robust basidiomata; fine, hyaline encrustations on the cheilocystidia, tramal hyphae and pileipellis hyphae; and absence of Pileocystidia.

\section{REFERENCES}

Hesler, L. R. and A. H. Smith, (1965). North American species of Crepidotus. Hafner Publishing Company, New York, U.S.A.

Kornerup, A. and J. H. Wanscher, (1978). Methuen Handbook of Colour. ( $3^{\text {rd }}$ edn.). Methuen, London, U.K.

Manjula, B. (1983). A revised list of the Agaricoid and boletoid basidiomycetes from India and Nepal. Proceedings of the Indian Academy of Sciences (Plant Sciences) 92: 81-213.

Mohanan, C. (2011). Macrofungi of Kerala. KFRI Handbook No. 27, Kerala Forest Research Institute, Peechi, Kerala, India

Natarajan, K. and N. Raman, (1981). South Indian Agaricales vii. Nova Hedwigia 34, 163-176.

Natarajan, K. and N. Raman, (1983). South Indian Agaricales. Bibliotheca Mycologica 89: 1-203.

Singer, R. (1986). The Agaricales in modern taxonomy. ( $4^{\text {th }}$ edn.). Koeltz Scientific Books, Koenigstein, Germany.

Vrinda, K. B., C. K. Pradeep, S. Mathew and T. K. Abraham, (2000). Some pleurotoid agarics from Western Ghats, Kerala. 